\title{
EXPRESSIONS OF SOLUTIONS OF ORDINARY DIFFERENTIAL EQUATIONS BY STANDARD FUNCTIONS
}

\author{
Z. NAVICKAS and L. BIKULČIENE் \\ Kaunas University of Technology \\ Studenty 50, Kaunas, Lithuania \\ E-mail: zenonas.navickas@ktu.lt; liepa.bikulciene@ktu.lt \\ Received October 15, 2005; revised September 18, 2006; published online December 15, 2006
}

\begin{abstract}
It is well known that solutions of many ordinary differential equations allow presentation in the power series form. In the paper we introduce and analyze rigorous conditions under which the later series (solutions) can be reduced to a finite sum of standard functions. Some areas of practical applicability of the proposed algorithm are discussed.
\end{abstract}

Key words: differential equations, algebraic progression, Van-der-Mond determinant, standard functions

\section{Introduction}

Various dynamical systems are described by ordinary differential equations. The power series solutions of these equations can be easily obtained using the operator method [1]. These series must be changed to polynomials with optional order in the case of a computer realization. It is possible to approximate those polynomials by a finite sum of exponential functions, provided solutions themselves are periodic or aperiodic functions, [2].

In the paper, representation of solutions in the form of a finite sum of standard functions is discussed. A new computational algorithm for discarding of non-significant terms, depending on accuracy prescribed, is presented. The developed approach has been successfully applied to solve a system of ordinary differential equations, used to describe reduction of wind or wave power into electrical energy. In the paper, the model has links with self-vibrations that are transformed into rotational motion. To say more, implementation of trigonometric functions not only facilitates evaluation of dynamical characteristics but also makes determination of the existence domains for steady modes more precise. 


\section{The General Part}

All the necessary notations, definitions and theorems associated with formulation of conditions, under which the power series solution of a differential equation can be reduced to a finite sum of standard functions, are presented below. In particular, the Newton's binomial coefficients and relevant expressions are to be used for $j, k \in Z_{0}$ :

$$
\left(\begin{array}{l}
j \\
k
\end{array}\right):=\left\{\begin{array}{ll}
0, & 0 \leq j<k, \\
\frac{j !}{k !(j-k) !}, & k \leq j,
\end{array} \quad\left(\begin{array}{l}
j \\
k
\end{array}\right) 0^{j-k}:=\left\{\begin{array}{l}
0, j \neq k, \\
1, k=j .
\end{array}\right.\right.
$$

\subsection{The Van-der-Mond determinants}

The Van-der-Mond determinant, as well as its generalized version, is introduced in this section. Let us note, that Van-der-Mond determinants are different from zero for all possible values of their elements.

Definition 1 . The set of numbers $\lambda_{1}, \lambda_{2}, \ldots, \lambda_{n} \in C, n \in N$, is called Vander-Mond set (i.e. $V$-set), provided $\lambda_{k} \neq \lambda_{r}$ for $k \neq r$.

Whatever the $V$-set, non-zero Van-der-Mond determinant can be written as:

$$
\begin{aligned}
V_{n}\left(\lambda_{1}, \lambda_{2}, \ldots, \lambda_{n}\right):= & \left|\begin{array}{ccccc}
1 & \lambda_{1} & \lambda_{1}^{2} & \ldots & \lambda_{1}^{n-1} \\
1 & \lambda_{2} & \lambda_{2}^{2} & \ldots & \lambda_{2}^{n-1} \\
\ldots & \ldots & \ldots & \ldots & \ldots \\
1 & \lambda_{n} & \lambda_{n}^{2} & \ldots & \lambda_{n}^{n-1}
\end{array}\right|=\left(\lambda_{2}-\lambda_{1}\right)\left(\lambda_{3}-\lambda_{1}\right)\left(\lambda_{3}-\lambda_{2}\right) \\
& \times \ldots .\left(\lambda_{n}-\lambda_{1}\right)\left(\lambda_{n}-\lambda_{2}\right) \ldots\left(\lambda_{n}-\lambda_{n-1}\right) \neq 0 . \quad(2.2)
\end{aligned}
$$

Obviously, $V_{1}\left(\lambda_{1}\right)=1$.

Let $\left\{\hat{\lambda}_{1}, \hat{\lambda}_{2}, \ldots, \hat{\lambda}_{m}\right\}$ be a $V$-set. Consider the following expressions:

$$
\begin{aligned}
& \Delta_{k}^{(0)}(j):=\hat{\lambda}_{k}^{j}, \quad \Delta_{k}^{(r+1)}(j):=\frac{\Delta_{k+1}^{(r)}(j)-\Delta_{k}^{(r)}(j)}{\hat{\lambda}_{k+r+1}-\hat{\lambda}_{k}}, \\
& r=0,1, \ldots ; \quad 1 \leq k \leq m-r-1 .
\end{aligned}
$$

It can be easily seen that these expressions (thoroughly described in [3]) are symmetric polynomials with the same degree terms in $\hat{\lambda}_{1}, \hat{\lambda}_{2}, \ldots, \hat{\lambda}_{m}$. For instance,

$$
\begin{aligned}
& \Delta_{k}^{(1)}(0):=0, \quad \Delta_{k}^{(1)}(j):=\frac{\hat{\lambda}_{k+1}^{j}-\hat{\lambda}_{k}^{j}}{\hat{\lambda}_{k+1}-\hat{\lambda}_{k}}=\sum_{\substack{k, l \in Z_{0}, k+l=j-1}}^{j-1} \hat{\lambda}_{k}^{k} \hat{\lambda}_{k+1}^{l}, \quad j=1,2,3, \ldots, \\
& \Delta_{k}^{(2)}(0)=\Delta_{k}^{(2)}(1)=0,
\end{aligned}
$$




$$
\Delta_{k}^{(2)}(j):=\frac{\frac{\hat{\lambda}_{k+2}^{j}-\hat{\lambda}_{k+1}^{j}}{\hat{\lambda}_{k+2}^{j}-\hat{\lambda}_{k+1}^{j}}-\frac{\hat{\lambda}_{k+1}^{j}-\hat{\lambda}_{k}^{j}}{\hat{\lambda}_{k+1}^{j}-\hat{\lambda}_{k}^{j}}}{\hat{\lambda}_{k+2}-\hat{\lambda}_{k}}=\sum_{\substack{k, l, r \in Z_{0} \\ k+l+r=j-2}}^{j-1} \hat{\lambda}_{k}^{k} \hat{\lambda}_{k+1}^{l} \hat{\lambda}_{k+2}^{r}, \quad j \geq 2 .
$$

On the basis of (2.1), (2.2) and (2.3), the following relationships are obtained:

$$
\begin{aligned}
& \Delta_{k}^{(0)}(j)=\frac{1}{V_{1}\left(\hat{\lambda}_{k}\right) \hat{\lambda}_{k}^{j}}, \quad \Delta_{k}^{(r+1)}(j):=\frac{1}{V_{r+2}\left(\hat{\lambda}_{k}, \hat{\lambda}_{k+1}, \ldots, \hat{\lambda}_{k+r+1}\right)}\left(\gamma_{r+1 r+1}\right. \\
& \quad \times V_{r+1}\left(\hat{\lambda}_{k}, \hat{\lambda}_{k+1}, \ldots, \hat{\lambda}_{k+r}\right) \hat{\lambda}_{k+r+1}^{j}+\gamma_{r+1 r} V_{r+1}\left(\hat{\lambda}_{k}, \ldots, \hat{\lambda}_{k+r-1}, \hat{\lambda}_{k+r+1}\right) \\
& \left.\quad \times \hat{\lambda}_{k+r}^{j}+\cdots+\gamma_{r+10} V_{r+1}\left(\hat{\lambda}_{k+1}, \hat{\lambda}_{k+2}, \ldots, \hat{\lambda}_{k+r+1}\right) \hat{\lambda}_{k}^{j}\right)
\end{aligned}
$$

where $\gamma_{r+1 l}=(-1)^{r+1+l}, \quad l=0,1, \ldots, r+1$.

For the coefficients $\gamma_{r+1 l}$, we have

$$
\gamma_{r+1 r+1} \gamma_{r+1 r} \cdots \gamma_{r+10}=(-1)\left(\begin{array}{c}
r+1 \\
2
\end{array}\right),
$$

where the Van-der-Mond determinants satisfy the relationship

$$
\begin{aligned}
& V_{r}\left(\hat{\lambda}_{k}, \hat{\lambda}_{k+1}, \ldots, \hat{\lambda}_{k+r-1}\right) V_{r}\left(\hat{\lambda}_{k}, \ldots, \hat{\lambda}_{k+r-2}, \hat{\lambda}_{k+r}\right) \ldots \\
& \quad \times V_{r}\left(\hat{\lambda}_{k+1}, \hat{\lambda}_{k+2}, \ldots, \hat{\lambda}_{k+r}\right)=V_{r+1}^{r-1}\left(\hat{\lambda}_{k}, \hat{\lambda}_{k+1}, \ldots, \hat{\lambda}_{k+r}\right), \quad r \geq 1 .
\end{aligned}
$$

For instance,

$$
\begin{aligned}
\Delta_{k}^{(1)}(j)= & \frac{1}{V_{2}\left(\hat{\lambda}_{k}, \hat{\lambda}_{k+1}\right)}\left(V_{1}\left(\hat{\lambda}_{k}\right) \hat{\lambda}_{k+1}^{j}-V_{1}\left(\hat{\lambda}_{k+1}\right) \hat{\lambda}_{k}^{j}\right), \\
\Delta_{k}^{(2)}(j)= & \frac{1}{\left(\hat{\lambda}_{k+2}-\hat{\lambda}_{k}\right)\left(\hat{\lambda}_{k+2}-\hat{\lambda}_{k+1}\right)} \hat{\lambda}_{k+2}^{j}-\frac{1}{\left(\hat{\lambda}_{k+1}-\hat{\lambda}_{k}\right)\left(\hat{\lambda}_{k+2}-\hat{\lambda}_{k+1}\right)} \hat{\lambda}_{k+1}^{j} \\
& \frac{1}{\left(\hat{\lambda}_{k+1}-\hat{\lambda}_{k}\right)\left(\hat{\lambda}_{k+2}-\hat{\lambda}_{k}\right)} \hat{\lambda}_{k}^{j}=\frac{1}{V_{3}\left(\hat{\lambda}_{1}, \hat{\lambda}_{2}, \hat{\lambda}_{3}\right)}\left(V_{2}\left(\hat{\lambda}_{k}, \hat{\lambda}_{k+1}\right) \hat{\lambda}_{k+2}^{j}\right. \\
& \left.-V_{2}\left(\hat{\lambda}_{k}, \hat{\lambda}_{k+2}\right) \hat{\lambda}_{k+1}^{j}+V_{2}\left(\hat{\lambda}_{k+1}, \hat{\lambda}_{k+2}\right) \hat{\lambda}_{k}^{j}\right), \quad j=0,1,2, \cdots .
\end{aligned}
$$

Since $\Delta_{j}^{(r)}$ are polynomials in $\hat{\lambda}_{1}, \hat{\lambda}_{2} \ldots, \hat{\lambda}_{m}$, the following limit is valid

$$
\lim _{\hat{\lambda}_{k}, \hat{\lambda}_{k+1}, \ldots, \hat{\lambda}_{k+r} \rightarrow \lambda_{k}} \Delta_{k}^{(r)}(j)=\left(\begin{array}{c}
j \\
r
\end{array}\right) \lambda_{k}^{j-r} .
$$

Now, let $\left\{\hat{\lambda}_{1}, \hat{\lambda}_{2}, \ldots, \hat{\lambda}_{n}\right\}$ and $\left\{\lambda_{1}, \lambda_{2}, \ldots, \lambda_{m}\right\}$ be two $V$-sets and $m_{1}, m_{2}$, $\ldots, m_{n}$ be natural numbers, satisfying the condition

$$
m_{1}+m_{2}+\ldots+m_{n}=m .
$$


Then, by using $\hat{\lambda}_{1}, \hat{\lambda}_{2}, \ldots, \hat{\lambda}_{m}$ and expressions $\Delta_{k}^{(r)}(j)$ we get the following form of the determinant

$$
\Phi_{m_{1}, m_{2}, \ldots, m_{n}}\left(\hat{\lambda}_{1}, \hat{\lambda}_{2}, \ldots, \hat{\lambda}_{m}\right):=\left|\begin{array}{cccc}
\Delta_{1}^{(0)}(0) & \Delta_{1}^{(0)}(1) & \ldots & \Delta_{1}^{(0)}(m-1) \\
\Delta_{1}^{(1)}(0) & \Delta_{1}^{(1)}(1) & \ldots & \Delta_{1}^{(1)}(m-1) \\
\ldots & \ldots & \ldots & \ldots \\
\Delta_{1}^{\left(m_{1}-1\right)}(0) & \Delta_{1}^{\left(m_{1}-1\right)}(1) & \ldots & \Delta_{1}^{\left(m_{1}-1\right)}(m-1) \\
\Delta_{m_{1}+1}^{(0)}(0) & \Delta_{m_{1}+1}^{(0)}(1) & \ldots & \Delta_{m_{1}+1}^{(0)}(m-1) \\
\ldots & \ldots & \ldots & \ldots \\
\Delta_{m_{1}+1}^{\left(m_{2}-1\right)}(0) & \Delta_{m_{1}+1}^{\left(m_{2}-1\right)}(1) & \ldots & \Delta_{m_{1}+1}^{\left(m_{2}-1\right)}(m-1) \\
\ldots & \ldots & \ldots & \ldots \\
D(0) & D(1) & \ldots & D(m-1)
\end{array}\right|
$$

here we use a notation

$$
D(j)=\Delta_{m_{1}+m_{2}+\ldots+m_{n-1}+1}^{\left(m_{n}-1\right)}(j)
$$

By using (2.4), it can be shown that

$$
\begin{aligned}
& \Phi_{m_{1}, m_{2}, \ldots, m_{n}}\left(\hat{\lambda}_{1}, \hat{\lambda}_{2}, \ldots, \hat{\lambda}_{m}\right)=V_{m}\left(\hat{\lambda}_{1}, \hat{\lambda}_{2}, \ldots, \hat{\lambda}_{m}\right) / V_{m_{1}}\left(\hat{\lambda}_{1}, \ldots, \hat{\lambda}_{m_{1}}\right) \\
& \times V_{m_{2}}\left(\hat{\lambda}_{m_{1}+1}, \ldots, \hat{\lambda}_{m_{1}+m_{2}}\right) \ldots \cdot V_{m_{n}}\left(\hat{\lambda}_{m_{1}+\ldots+m_{n-1}+1}, \ldots, \hat{\lambda}_{m}\right)
\end{aligned}
$$

Determinant $\Phi_{m_{1}, m_{2}, \ldots, m_{n}}\left(\hat{\lambda}_{1}, \hat{\lambda}_{2}, \ldots, \hat{\lambda}_{m}\right)$ is a polynomial (not necessarily symmetric) in $\hat{\lambda}_{1}, \hat{\lambda}_{2}, \ldots, \hat{\lambda}_{m}$; besides,

$$
\Phi_{m_{1}, m_{2}, \ldots, m_{n}}\left(\hat{\lambda}_{1}, \hat{\lambda}_{2}, \ldots, \hat{\lambda}_{m}\right) \neq 0 \text {. }
$$

Now, using the $V$-set $\left\{\lambda_{1}, \lambda_{2}, \ldots, \lambda_{n}\right\}$, we can construct the determinant

$$
\begin{aligned}
& \bar{V}_{m_{1}, m_{2}, \ldots, m_{n}}\left(\lambda_{1}, \lambda_{2}, \ldots, \lambda_{n}\right)=
\end{aligned}
$$

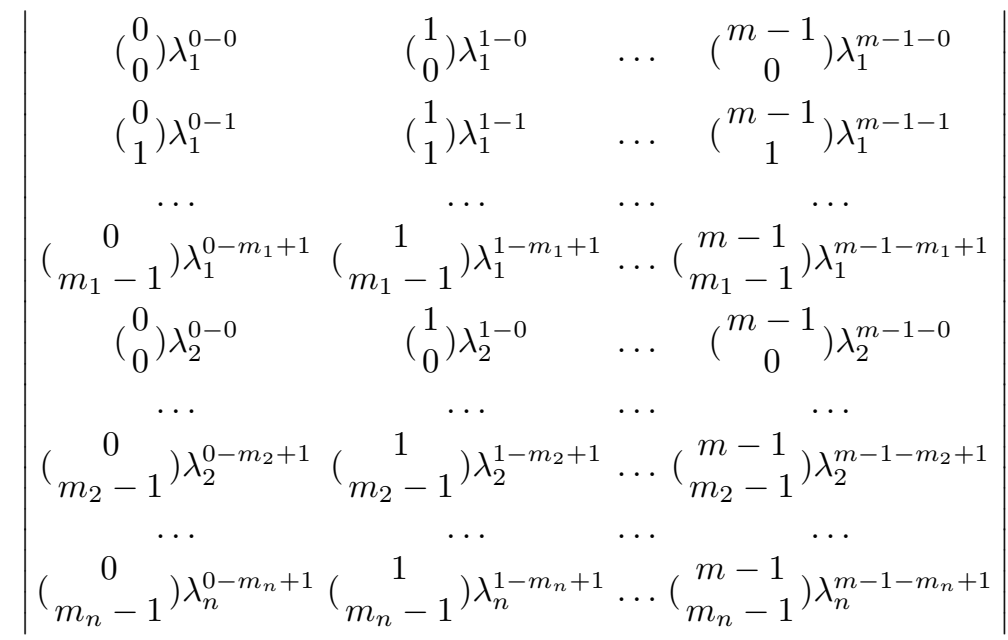


DeFinition 2. $\bar{V}_{m_{1}, m_{2}, \ldots, m_{n}}\left(\lambda_{1}, \lambda_{2}, \ldots, \lambda_{n}\right)$ is called the generalized Van-derMond determinant.

Equality (2.7) implies that

$$
\bar{V}_{m_{1}, m_{2}, \ldots, m_{n}}\left(\lambda_{1}, \lambda_{2}, \ldots, \lambda_{n}\right)=\lim _{\substack{\hat{\lambda}_{1}, \hat{\lambda}_{2}, \ldots, \hat{\lambda}_{m_{1} \rightarrow \lambda_{1}} \\ \hat{\lambda}_{m_{1}+1}, \ldots, \hat{\lambda}_{m_{1}+m_{2} \rightarrow \lambda_{2}} \\ \hat{\lambda}_{m_{1}+\ldots+m_{n-1}+\ldots, \hat{\lambda}_{m} \rightarrow \lambda_{n}}}} \Phi_{m_{1}, m_{2}, \ldots, m_{n}}\left(\hat{\lambda}_{1}, \hat{\lambda}_{2}, \ldots, \hat{\lambda}_{m}\right) .
$$

Consequently, the generalized Van-der-Mond determinant is a polynomial in $\lambda_{1}, \lambda_{2}, \ldots, \lambda_{n}$, besides, $\bar{V}_{m_{1}, m_{2}, \ldots, m_{n}}\left(\lambda_{1}, \lambda_{2}, \ldots, \lambda_{n}\right) \neq 0$.

We notice here that

$$
\bar{V}_{1,1, \ldots, 1}\left(\lambda_{1}, \lambda_{2}, \ldots, \lambda_{n}\right)=V_{n}\left(\lambda_{1}, \lambda_{2}, \ldots, \lambda_{n}\right), \quad \bar{V}_{n}\left(\lambda_{k}\right)=1,
$$

and so on.

Example 1. Given two $V$-sets $\left\{\hat{\lambda}_{1}, \hat{\lambda}_{2}, \hat{\lambda}_{3}\right\},\left\{\lambda_{1}, \lambda_{2}\right\}$, with $m_{1}=2, m_{2}=1$, then we easily derive:

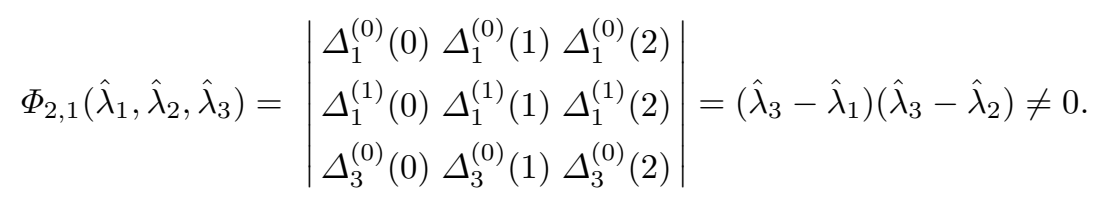

Then we get:

$$
\bar{V}_{2,1}\left(\lambda_{1}, \lambda_{2}\right)=\left|\begin{array}{ccc}
1 & \lambda_{1} & \lambda_{1}^{2} \\
0 & 1 & 2 \lambda_{1} \\
1 & \lambda_{2} & \lambda_{2}^{2}
\end{array}\right|=\left(\lambda_{2}-\lambda_{1}\right)^{2} \neq 0
$$

\subsection{The algebraic progressions}

In this section, the concept of an algebraic progression - generalization of an arithmetic/geometric progression - is presented. Some properties of the algebraic progressions are disclosed. Let a sequence of complex numbers $\left(p_{j} ; j \in Z\right)$ be given. Then, for every $m \in N$ and fixed $j_{0} \in Z_{0}$, it is possible to construct the Hankel matrix $H_{j_{0}}^{(m)}$ and the extended matrix $\bar{H}_{j_{0}}^{(m)}(\rho)$ (see [4]):

$$
\begin{aligned}
& H_{j_{0}}^{(m)}:=\left\|\begin{array}{cccc}
p_{j_{0}} & p_{j_{0}+1} & \ldots & p_{j_{0}+m-1} \\
p_{j_{0}+1} & p_{j_{0}+2} & \ldots & p_{j_{0}+m} \\
\ldots & \ldots & \ldots & \ldots \\
p_{j_{0}+m-1} & p_{j_{0}+m} & \ldots & p_{j_{0}+2 m-2}
\end{array}\right\|, \\
& \bar{H}_{j_{0}}^{(m)}(\rho):=\left\|\begin{array}{cccc}
p_{j_{0}} & p_{j_{0}+1} & \ldots & p_{j_{0}+m} \\
p_{j_{0}+1} & p_{j_{0}+2} & \ldots & p_{j_{0}+m+1} \\
\ldots & \ldots & \ldots & \ldots \\
p_{j_{0}+m-1} & p_{j_{0}+m} & \ldots & p_{j_{0}+2 m-1} \\
1 & \rho & \ldots & \rho^{m}
\end{array}\right\| .
\end{aligned}
$$


Definition 3. If for a sequence of complex numbers $\left(p_{j} ; j \in Z\right), r_{0}$ satisfies the condition $r_{0}=\max _{\substack{m \in N \\ j_{0} \in Z_{0}}} \operatorname{rank} H_{j_{0}}^{(m)}$, then the rank of the sequence of complex numbers $\left(p_{j} ; j \in Z\right)$ is equal to $r_{0}$.

Let us denote $H_{r}\left(p_{j} ; j \in Z\right)=r_{0}$.

Example 2. $H_{r}\left(a_{0}+j d ; j \in Z_{0}\right)=2$, provided $d \neq 0 . H_{r}\left(j^{2} ; j \in Z_{0}\right)=3$. It can be proved that $H_{r}\left(a_{l} j^{l}+a_{l-1} j^{l-1}+\ldots+a_{0} ; j \in Z_{0}\right)=l+1$, for $a_{l} \neq$ $0, l \in N$. Also, $H_{r}\left(a_{0}, a_{1}, \ldots a_{n}, 0,0, \ldots\right)=n+1$, for $a_{n} \neq 0$. Here we assume that $H_{r}(0,0, \ldots)=0$. The sequence $\left(j ! ; j \in Z_{0}\right)$ doesn't have $H_{r}$, because $\operatorname{det} H_{m}^{(j)} \neq 0$, for all $m \in N$ and $j \in Z_{0}$.

Some corollaries follow from the definition of $H_{r}$.

Corollary 1. Let $q_{j}=p_{j+n}$, where $n \in N$ is fixed and $m_{1}<m_{2}$. Then

$$
H_{r}\left(p_{j} ; j \in Z_{0}\right) \geq H_{r}\left(q_{j} ; j \in Z_{0}\right), \operatorname{rank} H_{j_{0}}^{\left(m_{1}\right)} \leq \operatorname{rank} H_{j_{0}}^{\left(m_{2}\right)} .
$$

Corollary 2. If $H_{r}\left(p_{j} ; j \in Z_{0}\right)=r_{0}$, then $\operatorname{det} H_{0}^{\left(r_{0}\right)} \neq 0$ and $\operatorname{det} H_{j}^{\left(r_{0}+1+n\right)} \equiv 0$, $j, n \in Z_{0}$. So, $H_{r}\left(p_{j} ; j \in Z_{0}\right)=\max _{m \in N} \operatorname{rank}_{0}^{(m)}$.

Corollary 3. The given sequence $\left(p_{j} ; j \in Z_{0}\right)$ has $H_{r}$, satisfying equality

$$
H_{r}\left(p_{j} ; j \in Z_{0}\right)=m, m \in Z_{0},
$$

if and only if constants $A_{0}, A_{1}, \ldots, A_{m-1} \in C$ are independent of $j$ and satisfy the condition

$$
A_{0} p_{j}+A_{1} p_{j+1}+\ldots+A_{m-1} p_{j+m-1}=p_{j+m}, \quad \forall j \in Z_{0} .
$$

Equality (2.8) makes no sense for $m^{\prime}<m$.

By taking $j=0,1, \ldots, m-1$ in (2.8) we get a system of linear equations, for finding all coefficients $A_{0}, A_{1}, \ldots, A_{m-1}$. The matrix of this system is equal to $H_{0}^{(m)}$ and $\operatorname{det} H_{0}^{(m)} \neq 0$. As example it is possible to prove that for sequence

$$
\left(a_{l} j^{l}+a_{l-1} j^{l-1}+\ldots+a_{0} ; j \in Z_{0}\right)
$$

the coefficients are defined as

$$
A_{r}=(-1)^{l-r}\left(\begin{array}{c}
l+1 \\
r
\end{array}\right), \quad r=0,1, \ldots, l .
$$

The correctness of this relationship follows directly from the combinatorial equality, [3]

$$
\sum_{r=0}^{l}(-1)^{l-r}\left(\begin{array}{c}
l+1 \\
r
\end{array}\right)(j+r)^{n}=(j+l)^{n}, \quad n=0,1, \ldots, l ; l \in N .
$$


Corollary 4. Two sequences of complex numbers, $\left(p_{j} ; j \in Z_{0}\right)$ and $\left(q_{j} ; j \in Z_{0}\right)$ are equal if and only if $H_{r}\left(p_{j} ; j \in Z_{0}\right)=H_{r}\left(q_{j} ; j \in Z_{0}\right)=m$ and $p_{j}=q_{j}$,for $j=0,1, \ldots, 2 m-1$.

Lemma 1. Let $\left(p_{j} ; j \in Z_{0}\right)$ be a sequence and

$$
p_{j}:=\sum_{r=1}^{m} \mu_{r} \lambda_{r}^{j}
$$

Let $\left\{\lambda_{1}, \ldots, \lambda_{m}\right\}$ be a $V$-set. Then we get the following equalities:

$$
\begin{aligned}
& \operatorname{det} H_{j}^{(m)}=\left(\mu_{1} \mu_{2} \cdots \mu_{m}\right)\left(\lambda_{1} \lambda_{2} \cdots \lambda_{m}\right)^{j} V_{m}^{2}\left(\lambda_{1}, \lambda_{2}, \cdots, \lambda_{m}\right), \\
& \operatorname{det} \bar{H}_{j}^{(m)}(\rho)=\operatorname{det} H_{j}^{(m)}\left(\rho-\lambda_{1}\right)\left(\rho-\lambda_{2}\right) \cdots\left(\rho-\lambda_{m}\right) .
\end{aligned}
$$

Proof. Really,

$$
\operatorname{det} H_{j}^{(m)}=\left(\mu_{1} \mu_{2} \cdots \mu_{m}\right)\left(\lambda_{1} \lambda_{2} \cdots \lambda_{m}\right)^{j} \sum_{\left(r_{1}, r_{2}, \ldots, r_{m}\right)} \operatorname{det}\left\|\lambda_{r_{k}}^{k+l-2}\right\|_{k, l=1}^{m},
$$

where summation is performed over all possible permutations $\left(r_{1}, r_{2}, \ldots, r_{m}\right)$ of integer numbers $1,2, \ldots, m$. Since

$$
\sum_{\left(r_{1}, r_{2}, \ldots, r_{m}\right)} \operatorname{det}\left\|\lambda_{r_{k}}^{k+l-2}\right\|_{k, l=1}^{m}=\left(\operatorname{det}\left\|\lambda_{k}^{l-1}\right\|\right)^{2}, \quad k, l=1, \ldots, m,
$$

the proof of identity (2.10) is obtained. The proof of identity (2.11) is carried out in a similar way.

Remark 1. For all $V$-sets $\left\{\lambda_{1}, \lambda_{2}, \ldots, \lambda_{n}\right\}$, the relationship $\left(\lambda_{1} \lambda_{2} \cdots \lambda_{m}\right)^{0}=1$ is valid.

Corollary 5. If $p_{j}$ is specified by (2.9), then $\operatorname{det} H_{j}^{(m)} \equiv 0$ for all $j \in Z_{0}$ and $n \geq m+1$. Therefore, $H_{r}\left(p_{j} ; j \in Z\right)=m$, provided $\mu_{1}, \mu_{2}, \ldots, \mu_{m} \neq 0$.

Now, using $V$-set $\left\{\lambda_{1}, \lambda_{2} \ldots, \lambda_{n}\right\}$, it is possible to form a sequence of numbers $\left(p_{j} ; j \in Z_{0}\right)$, which is given by

$$
p_{j}:=\sum_{r=1}^{n} \sum_{k_{r}=0}^{m_{r}-1} \mu_{r k_{r}}\left(\begin{array}{c}
j \\
k_{r}
\end{array}\right) \lambda_{r}^{j-k_{r}}, \quad m_{1}, m_{2}, \ldots, m_{n} \in N, \mu_{r k_{r}} \in C .
$$

Definition 4. The sequence of numbers, specified by (2.13), is called the algebraic progression, the coefficients $\lambda_{1}, \lambda_{2} \ldots, \lambda_{n}$ are said to be denominators of multiplicity $m_{1}, m_{2}, \ldots, m_{n}$, respectively, and $\mu_{r k_{r}}$ are called coefficients of the algebraic progression.

Remark 2. The sequences of numbers, proposed in the above examples and specified by (2.9), are algebraic progressions. Respective denominators can be found immediately. For instance, denominators of the sequence $\left(p_{j} ; j \in Z_{0}\right)$, $p_{j}=a_{0}+j d$, are given by $\lambda_{1,2}=1$, i.e., $p_{j}=a_{0} 1^{j}+d\left(\begin{array}{c}j \\ 1\end{array}\right) 1^{j-1}$. 
Thus, arithmetic and geometric progressions are algebraic progressions. Notice that the sequence

$$
\left(\Delta_{k}^{(r)}(j), j \in Z_{0}\right), \quad r=1,2, \ldots, m-k
$$

is an algebraic progression, besides,

$$
H_{r}\left(\Delta_{k}^{(r)}(j), j \in Z_{0}\right)=r+1
$$

For instance, by using identity (2.10), the limit calculus and relationships (2.1) we observe that $H_{r}$ of the sequence of numbers $\left(\left(\begin{array}{l}j \\ l\end{array}\right) \lambda_{k}^{j-l}, j \in Z_{0}\right)$ satisfies the relationship:

$$
H_{r}\left(\left(\begin{array}{l}
j \\
l
\end{array}\right) \lambda_{k}^{j-l}, j \in Z_{0}\right)=l+1
$$

besides,

$$
\operatorname{det} H_{j}^{(r+1)}=\left\{\begin{array}{l}
\left(\begin{array}{c}
l+1 \\
2
\end{array}\right) \\
(-1) \begin{array}{l}
(l+1) \cdot j \\
0, r \geq l+1 .
\end{array}, r=l,
\end{array}\right.
$$

Really, lemma 1 and relationships (2.5) and (2.6) imply that the determinant $\operatorname{det} H_{j}^{(l+1)}$ of the Hankel matrix $H_{j}^{(l+1)}$ for the algebraic sequence $\left(\Delta_{k}^{(r)}(j), j \in Z_{0}\right)$ can be expressed as follows:

$$
\begin{aligned}
& \operatorname{det} H_{j}^{(l+1)}=(-1)\left(\begin{array}{c}
l+1 \\
2
\end{array}\right) \frac{V_{l}\left(\hat{\lambda}_{k}, \ldots, \hat{\lambda}_{k+l-1}\right) \cdot \ldots \cdot V_{l}\left(\hat{\lambda}_{k+1}, \ldots, \hat{\lambda}_{k+l}\right)}{V_{l+1}^{l+1}\left(\hat{\lambda}_{k}, \ldots, \hat{\lambda}_{k+l}\right)} \\
& \quad \times\left(\hat{\lambda}_{k}, \ldots, \hat{\lambda}_{k+l}\right)^{j} \cdot V_{l+1}^{2}\left(\hat{\lambda}_{k}, \ldots, \hat{\lambda}_{k+l}\right)=(-1)\left(\begin{array}{c}
l+1 \\
2
\end{array}\right)_{\left(\hat{\lambda}_{k}, \ldots, \hat{\lambda}_{k+l}\right)^{j} .}
\end{aligned}
$$

Then,

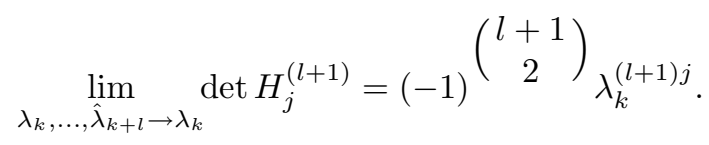

By taking into account properties of $\Delta_{k}^{(r)}(j)$ we state that expression (2.13) can be derived from (2.9) by passing to the limit:

$$
\begin{aligned}
& \lim _{\substack{\hat{\lambda}_{1}, \hat{\lambda}_{2}, \ldots, \hat{\lambda}_{m_{1} \rightarrow \lambda_{1}}, \hat{\lambda}_{m_{1}+1}, \ldots, \hat{\lambda}_{m_{1}+m_{2} \rightarrow \lambda_{2},}, \hat{\lambda}_{1} \hat{\lambda}_{m_{1}+\ldots+m_{n-1}+1, \ldots, \hat{\lambda}_{m} \rightarrow \lambda_{n}}}} \sum_{k_{1}=0}^{m_{1}-1} \mu_{1 k_{1}} \Delta_{1}^{\left(k_{1}\right)}(j)+\sum_{k_{2}=0}^{m_{2}-1} \mu_{2 k_{2}} \Delta_{m_{1}+1}^{\left(k_{2}\right)}(j)+\ldots \\
& \quad+\sum_{k_{n}=0}^{m_{n}-1} \mu_{n k_{n}} \Delta_{m_{1}+\ldots+m_{n-1}+1}^{\left(k_{n}\right)}(j)=\sum_{r=1}^{n} \sum_{k_{r}=0}^{m_{r}-1} \mu_{r k_{r}}\left(\begin{array}{c}
j \\
k_{r}
\end{array}\right) \lambda_{r}^{j-k_{r}}=p_{j},
\end{aligned}
$$

where $m_{1}+m_{2}+\ldots+m_{n}=m$; besides, $\left\{\lambda_{1}, \lambda_{2}, \ldots, \lambda_{n}\right\} \in C$ is a $V$-set. Then, $H_{r}\left(p_{j} ; j \in Z_{0}\right)=m$. 
Corollary 6 . If the $j$-th term $p_{j}$ of the algebraic progression $\left(p_{j} ; j \in Z_{0}\right)$ is described by (2.13), then Hankel matrices $H_{j}^{(m)}$ and $\bar{H}_{j}^{(m)}$ satisfy relationships:

$$
\begin{aligned}
\operatorname{det} H_{j}^{(m)} & =\sigma_{m}\left(\lambda_{1}^{m_{1}} \lambda_{2}^{m_{2}} \ldots \lambda_{n}^{m_{n}}\right)^{j}, \\
\operatorname{det} \bar{H}_{j}^{(m)} & =\operatorname{det} H_{j}^{(m)}\left(\rho-\lambda_{1}\right)^{m_{1}}\left(\rho-\lambda_{2}\right)^{m_{2}} \ldots\left(\rho-\lambda_{n}\right)^{m_{n}},
\end{aligned}
$$

provided $\sigma_{m}=\operatorname{det} H_{0}^{(m)} \neq 0$ and $\operatorname{det} H_{j}^{(m)}$ is independent of $j$, i.e.

$$
\begin{gathered}
\sigma_{m}=(-1)\left(\begin{array}{c}
m_{1} \\
2
\end{array}\right)+\left(\begin{array}{c}
m_{2} \\
2
\end{array}\right)+\ldots+\left(\begin{array}{c}
m_{n} \\
2
\end{array}\right){ }_{\mu_{1 m_{1}-1}^{m_{1}} \mu_{2 m_{2}-1}^{m_{2}} \ldots \mu_{n m_{n}-1}^{m_{n}}} \\
\times \bar{V}_{m_{1}, m_{2}, \ldots, m_{n}}^{2}\left(\lambda_{1}, \lambda_{2}, \ldots, \lambda_{n}\right) .
\end{gathered}
$$

Theorem 1. $H_{r}$ of the algebraic progression $\left(p_{j} ; j \in Z_{0}\right)$, specified by (2.13), satisfies the relationship:

$$
H_{r}\left(p_{j} ; j \in Z_{0}\right)=m_{1}+m_{2}+\ldots+m_{n}, \quad \text { where } \quad \mu_{r m_{r-1}} \neq 0 .
$$

The following two conclusions can be made.

Corollary 7. Let $\left(p_{j} ; j \in Z_{0}\right)$ and $\left(q_{j} ; j \in Z_{0}\right)$ be two algebraic progressions and $a, b \in C$ are fixed numbers. Then

$$
\left(a p_{j}+b q_{j} ; j \in Z_{0}\right), \quad\left(p_{j} q_{j} ; j \in Z_{0}\right), \quad\left(a p_{j}^{2}+b q_{j} ; j \in Z_{0}\right), \ldots
$$

are algebraic progressions and

$$
\begin{aligned}
& H_{r}\left(a p_{j}+b q_{j} ; j \in Z_{0}\right) \leq H_{r}\left(p_{j} ; j \in Z_{0}\right)+H_{r}\left(q_{j} ; j \in Z_{0}\right) ; \\
& H_{r}\left(p_{j} q_{j} ; j \in Z_{0}\right) \leq H_{r}\left(p_{j} ; j \in Z_{0}\right) H_{r}\left(q_{j} ; j \in Z_{0}\right) .
\end{aligned}
$$

Corollary 8. Let $H_{j}^{(m)}$ be Hankel matrices of algebraic progression $\left(p_{j} ; j \in Z_{0}\right)$. Then, (det $\left.H_{j}^{(m)} ; j \in Z_{0}\right)$ are algebraic progressions, for all $m \geq 1$.

Theorem 2. If a sequence of complex numbers $\left(p_{j} ; j \in Z_{0}\right)$ has

$$
H_{r}\left(p_{j} ; j \in Z_{0}\right)=m<+\infty,
$$

then this sequence is an algebraic progression.

Proof. First, using (2.8), a system of linear equations is formed. The matrix of this system is given by $H_{0}^{(m)}$, and $\operatorname{det} H_{0}^{(m)} \neq 0$. Then coefficients $A_{0}, A_{1}, \ldots, A_{m-1}$ are found. Expending the determinant $\operatorname{det} \bar{H}_{0}^{(m)}(\rho)$ with respect to the last row, we get

$$
\operatorname{det} \bar{H}_{0}^{(m)}(\rho)=\operatorname{det} H_{0}^{(m)}\left(A_{0}+A_{1} \rho+\ldots+A_{m-1} \rho^{m-1}-\rho^{m}\right) .
$$


Let roots $\lambda_{1}, \lambda_{2}, \ldots, \lambda_{n}$ of the algebraic equation $\bar{H}_{0}^{(m)}(\rho)=0$ be of multiplicity $m_{1}, m_{2}, \ldots, m_{n}$ respectively (here, $m_{1}+m_{2}+\ldots+m_{n}=m$ ). Then, from (2.13) we obtain

$$
\rho^{m+j}-A_{m-1} \rho^{m-1+j}-\ldots-A_{0} \rho^{j}=\rho^{j}\left(\rho-\lambda_{1}\right)^{m_{1}} \cdot \ldots \cdot\left(\rho-\lambda_{n}\right)^{m_{n}}, j \in Z_{0},
$$

and

$$
\left(\begin{array}{c}
j+m \\
k
\end{array}\right) \lambda_{r}^{m+j-k}=A_{m-1}\left(\begin{array}{c}
j+m-1 \\
k
\end{array}\right) \lambda_{r}^{m+j-1}+\ldots+A_{0}\left(\begin{array}{l}
j \\
k
\end{array}\right) \lambda_{r}^{m-k},
$$

for $r=1,2, \ldots, n, k=0,1, \ldots, m_{r}-1, j \in Z_{0}$. Since the determinant of the coefficient matrix of the constructed linear system

$$
\sum_{r=1}^{n} \sum_{k_{r}=0}^{m_{r}-1}\left(\begin{array}{c}
j \\
k_{r}
\end{array}\right) \lambda_{r}^{j-k_{r}} \mu_{r k_{r}}=p_{j}, \quad j=0,1, \ldots, m-1,
$$

represents a non-zero generalized Van-der-Mond determinant, there are no problems to find the coefficients $\mu_{r k_{r}}$ and to form the algebraic progression $\left(q_{j} ; j \in Z_{0}\right)$ described by the relationship

$$
q_{j}:=\sum_{r=1}^{n} \sum_{k_{r}=0}^{m_{r}-1} \mu_{r k_{r}}\left(\begin{array}{c}
j \\
k_{r}
\end{array}\right) \lambda_{r}^{j-k_{r}}, j \in N .
$$

The equality $q_{j}=p_{j}$ holds true for $j=0,1, \ldots, m-1$. Besides, taking successively $j=0,1, \ldots, m-1$, we find out

$$
\begin{aligned}
q_{j+m}= & \sum_{r=1}^{n} \sum_{k_{r}=0}^{m_{r}-1} \mu_{r k_{r}}\left(\begin{array}{c}
j+m \\
k_{r}
\end{array}\right) \lambda_{r}^{j+m-k_{r}} \\
= & A_{m-1}\left(\sum_{r=1}^{m} \sum_{k_{r}=0}^{m_{r}-1} \mu_{r k_{r}}\left(\begin{array}{c}
j+m-1 \\
k_{r}
\end{array}\right) \lambda_{r}^{j-k_{r}}\right)+\ldots \\
& +A_{0}\left(\sum_{r=1}^{m} \sum_{k_{r}=0}^{m_{r}-1} \mu_{r k_{r}}\left(\begin{array}{c}
j \\
k_{r}
\end{array}\right) \lambda_{r}^{j-k_{r}}\right)=A_{m-1} p_{j+m-1}+A_{0} p_{j}=p_{j+m} .
\end{aligned}
$$

Thus, $q_{j}=p_{j}$, for $j=m, \ldots, 2 m-1$. Now, the above corollaries imply that $p_{j}=q_{j}$, for $j \in Z_{0}$.

Remark 3. The proof of Theorem 2 gives us a computational algorithm for finding all denominators of the algebraic progression and coefficients of the progression.

\subsection{The algebraic functions}

In this section a special type of analytical functions (algebraic functions with finite rank) is described. The relationships between these functions and algebraic progressions is established. 
Let a power series of a real variable $x$ be given

$$
y(x)=\sum_{j=0}^{+\infty} p_{j} \frac{\left(x-x_{0}\right)^{j}}{j !}, \quad\left|p_{j}\right| \leq M^{j}, \quad 0 \leq M<\infty .
$$

This series is convergent for all values of $x, x_{0} \in R$ ( $x_{0}$ being fixed).

Definition 5. A function, specified by (2.14), is called an algebraic function with finite rank, if it can be represented as a finite sum:

$$
y(x)=\sum_{r=1}^{n} Q_{r}(x) e^{\lambda_{r} x},
$$

where

$$
Q_{r}(x)=\sum_{k_{r}=0}^{m_{r}-1} a_{r k_{r}} x^{k_{r}}, \quad m_{r} \geq 1, \quad a_{r k_{r}} \in C,
$$

besides, $a_{r m_{r}-1} \neq 0$.

Theorem 3. Power series (2.14) can be reduced to the form (2.15) if and only if coefficients $p_{j}$ in (2.14) represent an algebraic progression $\left(p_{j} ; j \in Z_{0}\right)$; besides, $H_{r}\left(p_{j} ; j \in Z_{0}\right)=m_{1}+m_{2}+\ldots+m_{n}$.

Proof. The proof is based on the following transformations:

$$
x^{l} e^{\lambda x}=x^{l} \sum_{j=0}^{+\infty} \frac{(\lambda x)^{j}}{j !}=(l !) \sum_{j=0}^{+\infty}\left(\begin{array}{l}
j \\
l
\end{array}\right) \lambda^{j-l} \frac{x^{j}}{j !} .
$$

Corollary 9. Algebraic function $y(x)$ (expression (2.15)) can be reduced to the form

$$
y(x)=\mu_{0}+\sum_{r=1}^{m}\left(\mu_{r} \cos \left(\lambda_{r}\left(x-x_{0}\right)\right)+\gamma_{r} \sin \left(\nu_{r}\left(x-x_{0}\right)\right)\right),
$$

if and only if the sequence $\left(p_{j} ; j \in Z_{0}\right)$ is an algebraic progression, satisfying relationships (2.9), besides, the real parts of its denominators are zeros.

Corollary 10. If sequence $\left(p_{j} ; j \in Z_{0}\right)$ is an algebraic sequence, then the series (2.14) is convergent, for all $x \in R$.

Based on the above theorems and corollaries, the following conclusion can be made.

Corollary 11. Let function $y(x)$ be specified by power series (2.14) and it satisfies relationships (2.15). Then sequence

$$
\left(\hat{p}_{k} ; k \in Z_{0}\right), \quad \hat{p}_{k}:=y\left(k h+x_{0}\right), \quad h, x_{0} \in R
$$


is an algebraic progression with denominators $\Lambda_{r}=e^{h \lambda_{r}}, r=1, \ldots, n$, if $\lambda_{r}$ are the denominators of algebraic progression $\left(p_{j} ; j \in Z_{0}\right)$ and they compose a $V$-set. In general case a set $\left\{\Lambda_{1}, \Lambda_{2}, \ldots, \Lambda_{n}\right\}$ is not a $V$-set, i.e. some $\Lambda_{r_{1}}$, $\Lambda_{r_{2}}$ can satisfy the relationship $\Lambda_{r_{1}}=\Lambda_{r_{2}}$, when $r_{1} \neq r_{2}$, besides, $\Lambda_{r} \neq 0, r=$ $1,2, \ldots, n$. As a result, we get

$$
H_{r}\left(\hat{p}_{k} ; k \in Z_{0}\right) \leq H_{r}\left(p_{j} ; j \in Z_{0}\right) .
$$

\section{Applications}

Let a solution of a differential equation is given, i.e.:

$$
f(t)=\sum_{k=0}^{+\infty} p_{k} \frac{t^{k}}{k !}, \quad k=0,1,2, \ldots, t \in R .
$$

We are interested in representing this solution as a finite sum of exponential functions, namely:

$$
f(t)=\sum_{r=1}^{m} \mu_{r} e^{\lambda_{r} t}
$$

The following fact is taken into consideration

$$
\max \left(l: l \in N, \quad \operatorname{det} H_{0}^{(m)} \neq 0\right)=m .
$$

Next, by using some methods of linear algebra, we find coefficients $\mu_{r}$ and $\lambda_{r}$ in expression (3.1) from

$$
\operatorname{det} \bar{H}_{0}^{(m)}(\rho)=0, \quad p_{k}=\sum_{r=0}^{m} \lambda_{r}^{k} \mu_{r}, \quad k=0,1,2, \ldots, m-1 .
$$

Suppose, $\rho_{1}, \rho_{2}, \ldots, \rho_{m}$ is $V$-set. Then, $\rho_{k}=\lambda_{k}, k=1,2, \ldots, m$.

Example 3. Let

$$
y(x)=2 \sum_{k=0}^{+\infty} 3^{k-1} \frac{x^{2 k}}{(2 k) !}+\sum_{k=0}^{+\infty} 3^{k} \frac{x^{2 k+1}}{(2 k+1) !}
$$

be a solution of a differential equation. Obviously, the power series coefficients are:

$$
p_{j}=\left\{\begin{array}{l}
2 \cdot 3^{k-1}, j=2 k, \\
3^{k}, j=2 k+1,
\end{array} \quad k=0,1,2 \ldots\right.
$$

and $H_{r}\left(p_{j} ; j \in Z_{0}\right)=2$. Condition (3.2) implies

$$
\left|\begin{array}{ccc}
p_{0} & p_{1} & p_{2} \\
p_{1} & p_{2} & p_{3} \\
1 & \rho & \rho^{2}
\end{array}\right|=-\left(\rho^{2}-3\right)=0, \quad \lambda_{1}=\sqrt{3}, \quad \lambda_{2}=-\sqrt{3},
$$


i.e.

$$
y(x)=\mu_{1} e^{\sqrt{3} x}+\mu_{2} e^{-\sqrt{3} x}, \quad\left\{\begin{array}{l}
\mu_{1}+\mu_{2}=2 / 3 \\
\mu_{1} \sqrt{3}-\mu_{2} \sqrt{3}=1 .
\end{array}\right.
$$

Thus,

$$
\mu_{1,2}=\frac{2 \pm \sqrt{3}}{6}, \quad y(x)=\frac{2+\sqrt{3}}{6} e^{\sqrt{3} x}+\frac{2-\sqrt{3}}{6} e^{-\sqrt{3} x} .
$$

Example 4. Let a system of ordinary differential equations

$$
\left\{\begin{array}{l}
l \varphi^{\prime \prime}-x^{\prime \prime} \sin \varphi+H \varphi^{\prime}=0 \\
x^{\prime \prime}+p^{2} x-q_{1} x^{\prime}+q_{3} x^{\prime 3}-\mu\left(\varphi^{\prime \prime} \sin \varphi+\varphi^{\prime 2} \cos \varphi\right)=0
\end{array}\right.
$$

be given. Here $H, p, q_{1}, q_{3}, \mu$ are parameters, whose numerical values should be chosen to provide autonomous vibration $x(t)$ and to stimulate rotational motion $\varphi=\varphi(t)$. In particular, $H$ is a dissipative coefficient, $p$ stands for the self frequency of the system, $q_{1}$ and $q_{3}$ are self-vibration parameters, $\mu$ express the mass of rotor.

One of the main steady modes of the system is characterized by the uniform rotation of the rotor. For instance, such a mode is ensured if the parameter values are chosen to be

$$
H=0.05, \quad \mu=0.1, \quad p=1, \quad q_{1}=0.5, \quad q_{3}=1.75
$$

and initial conditions

$$
x(0)=x^{\prime}(0)=0.1, \quad \varphi(0)=0.1, \quad \varphi^{\prime}(0)=1 .
$$

By applying the operator method we get the following result (see [1])

$$
\begin{aligned}
\varphi^{\prime}(t)=0.7678+0.0531 t & +0.2877 t^{2}-0.0309 t^{3}-0.0839 t^{4} \\
& +0.0054 t^{5}+0.0098 t^{6}-\ldots
\end{aligned}
$$

By using the adapted operator method, we obtained a finite sum of trigonometric functions

$$
\tilde{\varphi}^{\prime}(t)=0.9324+0.1646 \cos 1.87 t-0.0283 \sin 1.87 t .
$$

The accuracy of the approximation is up to four decimal places.

Since the proposed method discloses influence of higher harmonics to the solutions obtained, it appears to be easier to find out other dynamical characteristics and existence domains for stable modes.

Example 5. Let the function

$$
\begin{aligned}
y(x)=\sum_{j=0}^{+\infty} p_{j} \frac{x^{j}}{j !} & =0.093 e^{x}-7^{0.2} e^{-0.2 x}-20.124 e^{2.13 x}+5^{\frac{1}{12}} e^{2^{\frac{7}{20}} x} \\
& -\cos (\sqrt{2}) e^{-0.1 e^{\sqrt{2}} x}-0.082 e^{0.06 x}+\sqrt{7} e^{-0.7 x} \\
& +0.175 e^{0.801 x}+\frac{1}{2} \pi e^{-0.914 x}+\sqrt{2} \sqrt{\pi} e^{-2.18 \sqrt{5} x}
\end{aligned}
$$


be a solution of a certain differential equation.

In this case $H_{r}\left(p_{j} ; j \in Z_{0}\right)=11$. Let us suppose that only the values of this function $y(0.4 k)=\hat{p}_{k}, k \in Z_{0}$ are known. The determinants $\operatorname{det} H_{0}^{(k)}$ of matrices $H_{0}^{(k)}, k=0,1,2, \ldots$ which are composed using sequence $\left(\hat{p}_{k} ; k \in Z_{0}\right)$ vanish monotonically. Then it is estimated that $\operatorname{det} H_{0}^{(6)}:=0$, as really $\operatorname{det} H_{0}^{(6)} \approx 10^{-5}$. The estimation $y_{0}(x)$ of function $y(x)$ is computed:

$$
\begin{aligned}
y_{0}(x)=\sum_{j=0}^{+\infty} q_{j} \frac{x^{j}}{j !}=2.51 e^{-4.88 x}+3.67 e^{-0.83 x}- & 20.65 e^{0.3 x} \\
+ & 0.55 e^{1.19 x}+0.62 e^{1.31 x} .
\end{aligned}
$$

It is noticed that $H_{r}\left(q_{j} ; j \in Z_{0}\right)=5$, besides,

$$
\left|y_{0}(x)-y(x)\right|<10^{-6}, \quad x \in[-1,1] .
$$

\section{Conclusions}

Some new concepts, associated with functions and sequences of series coefficients, are introduced. Conditions for the representation of power series as a finite sum of standard functions are formulated. Usable expressions and computational simplifications for the coefficients of a dynamical system are developed. The method can be applied to both the detection of existence domains for the solutions of stable differential equations and the analysis of the influence of higher harmonics to the type of the solution.

\section{References}

[1] L. Bikulčienè, R. Marcinkevičius and Z. Navickas. Computer realization of the operator method for solving of differential equations. In: Lubin Vulkov Zhilin Li and Jerzy Waśnewski(Eds.), Numerical Analysis and Its Applications. Third International Conference, NAA 2004, Lecture Notes in Computer Science, Springer-Verlag, Berlin, 182-189, 2005.

[2] L. Bikulčienė and Z. Navickas. Solution of differential equations using sum of exponential functions. Lithuanian Mathematical Journal, 44(1), 138-143, 2004.

[3] J.Riodan. Combinatorial Identities. New York-London-Sydney, 1968. (in English)

[4] R. Zaare-Nahandi and H. Usefi. A note of minors of a generalized Hankel matrix. International Math. Journal, 3(11), 1197-1201, 2003. 